\title{
Upping the anti
}

\author{
The fledgling field of antiferromagnetic spintronics looks set to bring exotic forms of magnetism into the realm \\ of practical applications.
}

P hysics undergraduates the world over learn about antiferromagnetism as a curiosity. The form of magnetism that mesmerizes us as children - if nothing else, as objects that stick to our fridges - is ferromagnetism. The fact that in some magnets the constituent spins orient themselves in alternating directions, thus resulting in a zero net magnetization, possibly even comes as a bit of a disappointment: antiferromagnets are like magnets, but macroscopically they don't display any of the properties we associate with magnetism. And so they don't stick to fridges. Great.

Beneath the surface, however, there is much more to antiferromagnets than meets the eye. Although it was Louis Néel who originally pointed out that there could be systems whose energy is minimized when their spins align in an antiparallel fashion $^{1}$, it took the power of a local probe, neutron scattering, to uncover this magnetic structure experimentally ${ }^{2}$. Over the decades, it has become apparent that between the two limiting regimes of fully ordered (ferromagnetic) and fully antiparallel (antiferromagnetic) ground states there exist a rich variety of 'intermediate' magnetic structures: these include highly degenerate, frustrated phases ${ }^{3}$ and modulated spin spirals and helices that, with the appropriate symmetries and balance of energy scales, can also be organized into more complex emergent structures such as magnetic skyrmions $^{4}$ and vortices ${ }^{5}$.

In spite of the fascinating physics they give rise to, controlling the properties of antiferromagnets, let alone manipulating them for practical applications, has been difficult precisely because of their imperviousness to external magnetic fields. But for a few exceptions as passive elements used to pin or 'harden' ferromagnetic layers, antiferromagnets have found little use in information storage technologies. Spintronics, the field of study that deals with the understanding and exploitation of electron spins and their transport in solid-state devices, has therefore traditionally focused on ferromagnets.

However, with the development of approaches to detect and control magnetism using electric currents, attention has recently started to shift towards antiferromagnets. In particular, following theoretical proposals to use spin-orbit interactions as a means to generate current-induced internal fields whose sign alternates with the periodicity of an antiferromagnetic lattice ${ }^{6}$, an important experimental breakthrough was made in 2016, with the demonstration of electrical switching between stable antiferromagnetic configurations in CuMnAs thin-film devices ${ }^{7}$. All of a sudden, the lack of magnetic moment and concomitant insensitivity of antiferromagnets to fields became an advantage: any stored magnetic state is insensitive to and produces no external magnetic field perturbations. Antiferromagnetic spintronics has become an experimental reality.

In this issue we explore the developments that have been made in the past few years regarding the spin transport properties of antiferromagnetic materials. As Tomas Jungwirth and colleagues note in their Comment on page 200, one of the major attractions is the prospect of efficient magnetic switching at speeds far in excess of those possible with ferromagnets and semiconductors. But looking further ahead, antiferromagnets also hold promise for technologies such as magnetic random access memories, neuromorphic computing and $\mathrm{THz}$ information technologies.

Of course, in spite of their new-found advantages and practical potential, the spin dynamics of antiferromagnets are more complex than their ferromagnetic counterparts, an issue considered by Olena Gomonay and co-workers in their Perspective on page 213. The conditions required to drive spin textures and excitations such as domain walls, skyrmions and spin waves in antiferromagnets are also different from those that we are intuitively familiar with in ferromagnets. So while there are definite opportunities and new functionalities afforded by spin transport and spin torque phenomena in these systems (see also the Review by Jakub Železný and colleagues on page 220), a reappraisal of the effects they give rise to is needed - it is not sufficient to simply make a direct mapping from ferromagnetism.

In another Perspective on page 217, Rembert Duine et al. focus on synthetic antiferromagnets consisting of anti-aligned stacks of ferromagnetic multilayers. As their behaviour is dictated by a competition of length scales, these can be thought of as materials with properties in between those of ferromagnets and antiferromagnets. Exploiting such tunable characteristics is another avenue for further enquiry. One such approach, which has in fact been extended to more conventional antiferromagnets as well, is ultrafast magnetic switching using lasers. Such optomagnetic control techniques also permit access to higher frequency excitations (such as magnons) in the $\mathrm{THz}$ regime, as Petr Němec and colleagues review on page 229 .

Finally, Libor Šmejkal and co-workers survey the links between antiferromagnetic spintronics and topologically protected electronic states that have garnered so much attention in condensed-matter physics over the past decade. While proposals for realizing Majorana and Weyl fermions in antiferromagnetic systems have, so far, only been made theoretically, several compensated non-collinear antiferromagnets with strong spin-orbit coupling have been shown to display experimentally a large anomalous Hall effect even in the absence of a net magnetization. It therefore seems already clear that the intersection between antiferromagnetism and topology will be a rich vein of research.

In covering the recent developments of this emerging field, it is apparent that so far the emphasis has been on fundamental effects - we are not about to see an antiferromagnetic memory device entering the market soon. But it should be equally clear that the potential to create a platform for a highly efficient category of magnetic control and information storage. Not bad for a class of magnets we used to view as a mere curiosity.

Published online: 2 March 2018 https://doi.org/10.1038/s41567-018-0091-2

\footnotetext{
References

1. Néel, L. Ann. Phys. 10, 5-105 (1932).

Shull, C. G. \& Smart, J. S. Phys. Rev. 76, 1256-1257 (1949).

3. Moessner, R. \& Ramirez, A. P. Phys. Today 59, 24-29 (February 2006).

4. Mühlbauer, S. et al. Science 323, 915-919 (2009).

5. Gao, S. et al. Nat. Phys. 13, 157-161 (2017).

6. Železný, J. et al. Phys. Rev. Lett. 113, 157201 (2014).

7. Wadley, P. et al. Science 351, 587-590 (2016).
} 